

О. Ю. Шведов, О перенормировке моделей типа Ли в пространстве произвольной размерности, Матем. заметки, 2000, том 68, выпуск 1, 154-156

DOI: https://doi.org/10.4213/mzm933

Использование Общероссийского математического портала Math-Net.Ru подразумевает, что вы прочитали и согласны с пользовательским соглашением http://www . mathnet.ru/rus/agreement

Параметры загрузки:

IP : 54.197 .130 .99

26 апреля 2023 г., 16:23:06

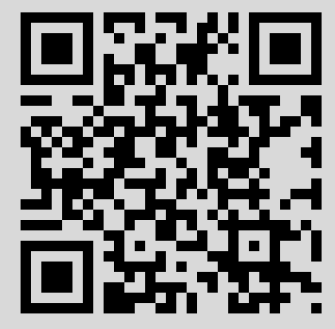




\section{О ПЕРЕНОРМИРОВКЕ МОДЕЛЕЙ ТИПА ЛИ В ПРОСТРАНСТВЕ ПРОИЗВОЛЬНОЙ РАЗМЕРНОСТИ}

\section{О. Ю. Шведов}

В квантовой теории поля возникает проблема перенормировки, заключающаяся в придании математического смысла расходящимся выражениям [1]. Для релятивистских локальных теорий в четырехмерном пространстве-времени данная проблема решена только в рамках теории возмущений. В этой связи представляет интерес исследование простых точно решаемых моделей [2], [3] на которых проблема перенормировок изучается до конца. Можно ожидать, что методы перенормировок, применяемые в точно решаемых моделях, будут применимы и к локальньм теориям поля.

В данном сообщении рассматривается модель Ли [3]-[5], возникающая в задачах ядерной физики. В одномерном и двумерном случаях данная модель может быть математически построена с помошью метода самосопряженных расширений операторов в гильбертовом пространстве [2], [6], в трехмерном случае - с помощью аналогичного метода в пространстве с индефинитной метрикой [3]. В пространстве более высоких размерностей математическое построение модели методами [3], [7] невозможно. По классификации [6] билинейная форма, отвечающая гамильтониану взаимодействия модели, является $(d-1)$-сингулярной в $d$-мерном случае; развитые в [6] методы применимы только к $s$-сингулярньг билинейным формам при $s=0,1$.

Для построения перенормированной модели в сообщении используется метод, основанньй на концепции, рассмотренной в [8]. Вместо исходного уравнения, не имеющего математического смысла, рассматривается регуляризованное уравнение, зависящее от малого параметра; при необходимости в уравнение добавляются контрчлены. После этого накладываются определенные требования на зависимость от малого параметра как входящих в уравнение коэффициентов, так и начального условия. При этом условие на начальное данное должно быть инвариантно относительно эволюции. Класс начальных данных, удовлетворяющих поставленным условиям, играет роль пространства состояний.

Конкретньй вид условий на начальные данные может быть получен, например, с помощью процедуры Боголюбова [1], основанной на плавном включении и выключения взаимодействия.

В модели Ли состояния задаются наборами $\left(c_{t}, \varphi_{t}(\boldsymbol{k})\right)$, состоящими из комплексного числа $c$, играющего роль амплитуды вероятности того, что в системе находится одна частица, и комплексной функции $\varphi(\boldsymbol{k})$, являющейся амплитудой вероятности того, что имеются две частицы с импульсами $\boldsymbol{k}$ и $\boldsymbol{- k}$. Для описания эволюции системы рассматривается следующая система уравнений:

$$
\begin{aligned}
i \frac{d c}{d t} & =g \int d \boldsymbol{k} \xi(\boldsymbol{k}) \varphi(\boldsymbol{k}), \\
i \frac{\partial \varphi}{\partial t} & =\omega \varphi+g c \xi
\end{aligned}
$$

где $\omega$ - оператор умножения на функцию $\omega(\boldsymbol{k})=\sqrt{\boldsymbol{k}^{2}+\mu^{2}}, g, \mu \in \mathbb{R}, \boldsymbol{k} \in \mathbb{R}^{d}, \xi(\boldsymbol{k})=\omega(\boldsymbol{k})^{-1 / 2}$, аргумент $t$ у функций $\varphi$ и $c$ опущен. Ввиду ульрафиолетовых расходимостей, связанных с недостаточно быстрым убыванием функции $\xi(\boldsymbol{k})$ при $\boldsymbol{k} \rightarrow \infty$ система (1) не имеет смысла.

Для придания системе (1) математического смысла рассмотрим процедуру регуляризации и перенормировки. Пусть $a$ - устремляемый в дальнейшем к нулю параметр регуляризации. Заменим функцию $\xi$ на быстро убьвающую при $\boldsymbol{k} \rightarrow \infty$ функцию $\xi^{a}$, которая при $a \rightarrow 0$ стремится к $\xi$. K правой части первого уравнения системы (1) добавим контрчлен $\sum_{l=0}^{m} i^{l} z_{l} c^{(l)}$, где $c^{(l)} \equiv \frac{d^{l}}{d t^{l}} c$ :

$$
\begin{aligned}
& i \frac{d c^{a}}{d t}=g\left(\xi^{a}, \varphi^{a}\right)+\sum_{l=0}^{m} i^{l} z_{l}^{a} c^{a(l)}, \\
& i \frac{\partial \varphi^{a}}{\partial t}=\omega \varphi^{a}+g c^{a} \xi^{a} .
\end{aligned}
$$

Найдем, при какой зависимости контрчленов $z_{l}$ от параметра регуляризации $a$ система $(2)$ будет иметь смысл и при $a \rightarrow 0$. Исследуем систему (2) при зависящем от $t$ коэффициенте $g=g_{t}$, которьй 
обращается в нуль вне некоторого интервала. После замены $\varphi_{t}=e^{-i \omega t} \phi_{t}$ система (2) принимает вид

$$
\begin{gathered}
\phi_{t}^{a}=\phi_{-\infty}^{a}-i \sum_{l=0}^{m-1}(-1)^{n}\left(g_{t} c\right)^{(n)} \frac{e^{i \omega t}}{(i \omega)^{l+1}} \xi^{a}-i(-1)^{m} \int_{-\infty}^{t} d \tau \frac{e^{i \omega \tau}}{(i \omega)^{m}}\left(g_{\tau} c_{\tau}^{a}\right)^{(m)} \xi^{a}, \\
g_{t}\left(\xi^{a}, e^{-i \omega t} \phi_{-\infty}^{a}\right)+\sum_{l=0}^{m} i^{l} \widetilde{z}_{l}^{a} c_{t}^{a(l)}+i^{m-1} g_{t} \int_{-\infty}^{t} d \tau\left(\xi^{a}, \frac{e^{-i \omega(t-\tau)}}{\omega^{m}}\left(g_{\tau} c_{\tau}^{a}\right)^{(m)} \xi^{a}\right)=0 .
\end{gathered}
$$

Через $\widetilde{z}_{l}^{a}$ обозначены коэффициенты, определяемые из соотношения

$$
\sum_{l=0}^{m} i^{l} z_{l}^{a} \frac{d^{l}}{d t^{l}}-i \frac{d}{d t}-\sum_{l=0}^{m-1} i^{l} g(t)\left(\xi^{a}, \frac{1}{\omega^{l+1}} \xi^{a}\right) \frac{d^{l}}{d t^{l}} g(t)=\sum_{l=0}^{m} i^{l} \widetilde{z}_{l}^{a} \frac{d^{l}}{d t^{l}}
$$

Система (3) регулярна при $a \rightarrow 0$, если коэффициенты $\widetilde{z}_{l}^{a}$ имеют предел при $a \rightarrow 0$.

Tеорема 1. Пусть $z_{m}^{a} \geqslant C_{1}>0, \omega \geqslant C_{2}>0$, не зависящая от а функция $\phi_{-\infty}(\boldsymbol{k})$ принадлехит пространству Шварца, $\left(\xi, \omega^{-m} \xi\right)<\infty u\left\|\xi^{a} / \omega^{m / 2}-\xi / \omega^{m / 2}\right\|_{L^{2}} \rightarrow 0$. Тогда при $а \rightarrow 0$

$$
c^{a(l)} \rightarrow c^{(l)}, \quad l=0, \ldots, m-1,
$$

а функиия $\widetilde{\varphi}^{a}$, определяемая из соотношения

$$
\varphi^{a}=-\sum_{l=0}^{m-1} i^{l}\left(g c^{a}\right)^{(l)} \frac{1}{\omega^{l+1}} \xi^{a}+\widetilde{\varphi}^{a},
$$

удовлетворяет свойству

$$
\left\|\omega^{m / 2} \widetilde{\varphi}^{a}-\omega^{m / 2} \widetilde{\varphi}\right\|_{L^{2}} \rightarrow 0 .
$$

В формулах (4), (6) сходимость является равномерной на интервале $t \in\left(t_{1}, t_{2}\right)$.

Оказьвается, что условия на решение системы (2), полученные в теореме 1 , инвариантны относительно эволюции.

Теорема 2. Пусть в предположениях теоремь 1 начальное условие для системь (2) при $t=0$ удовлетворяет условиям (4), (6). Тогда решение системы (2) также удовлетворяет этому условию.

ЗАмечАния. 1. Утверждения, аналогичные теоремам 1 и 2 , справедливы и при более слабом предположении $\left(\xi, \omega^{-(m+1)} \xi\right)<\infty,\left\|\xi^{a} / \omega^{(m+1) / 2}-\xi / \omega^{(m+1) / 2}\right\|_{L^{2}} \rightarrow 0$, если выполнено условие полярности ядра интегрального уравнения:

$$
\left(\xi^{a}, \frac{e^{-i \omega t}}{\omega^{(m+1)}} \xi^{a}\right) t^{\alpha} \leqslant C_{3}, \quad 0<\alpha<1
$$

В этом случае вместо (6) справедливо свойство

$$
\left\|\omega^{(m+1) / 2} \widetilde{\varphi}^{a}-\omega^{(m+1) / 2} \widetilde{\varphi}\right\|_{L^{2}} \rightarrow 0
$$

2. При $z_{m}=0$ в предположениях предыдущего замечания утверждение, аналогичное теореме 2, справедливо при $g \neq 0$. Доказательство данного утверждения вытекает из общей теории интегральных уравнений Вольтерра первого рода с сингулярным ядром [9].

3. Если условие (6) на начальне данное не выполнено, условие на решение $\varphi_{t}$ имеет более сложньй вид. В частности, при $a \rightarrow 0$ в главном порядке по $1 /|\boldsymbol{k}|$ имеем

$$
\varphi_{t}=-\frac{g_{t} c_{t}}{\omega} \xi+e^{-i \omega t}\left(\varphi_{0}+\frac{g_{0} c_{0}}{\omega} \xi\right) .
$$


Рассмотрим аналог скалярного произведения в данной модели. Обозначим

$$
\begin{gathered}
\psi^{a}=\left(c^{a}, \dot{c}^{a}, \ldots, c^{a(m-1)}, \varphi^{a}\right), \\
\left\langle\psi^{a}, \psi^{a}\right\rangle=\left(\varphi^{a}, \varphi^{a}\right)-\sum_{\substack{j, l \\
j+l+1 \leqslant m}} i^{l-j} \widetilde{z}_{j+l+1} c^{a *(j)} c^{a(l)} \\
-\sum_{\substack{j, l \\
j+l+1 \leqslant m-1}} i^{l-j}\left(\xi^{a}, \frac{1}{\omega^{j+l+2}} \xi^{a}\right)\left(g c^{a *}\right)^{(j)}\left(g c^{a}\right)^{(l)} .
\end{gathered}
$$

Данное скалярное произведение может быть представлено в виде

$$
\begin{aligned}
& \left\langle\psi^{a}, \psi^{a}\right\rangle \rightarrow\left(\widetilde{\varphi}^{a}, \widetilde{\varphi}^{a}\right)-\sum_{l=0}^{m-1} i^{l}\left(g c^{a}\right)^{(l)}\left(\widetilde{\varphi}^{a}, \frac{1}{\omega^{l+1}} \xi^{a}\right) \\
& -\sum_{j=0}^{m-1}(-i)^{j}\left(g c^{a *}\right)^{(j)}\left(\xi^{a}, \frac{1}{\omega^{j+1}} \widetilde{\varphi}^{a}\right)+\sum_{\substack{j, l \\
j+l+1 \geqslant m \\
, l=0, \ldots, m-1}} i^{l-j}\left(\xi^{a}, \frac{1}{\omega^{j+l+2}} \xi^{a}\right)\left(g c^{a *}\right)^{(j)}\left(g c^{a}\right)^{(l)} \\
& -\sum_{\substack{j, l \\
j=l+1 \leqslant m}} i^{l-j} \widetilde{z}_{j+l+1}^{a} c^{a *(j)} c^{a(l)} .
\end{aligned}
$$

Теорема 3. 1. СКалярное произведение (8) сохраняется во времени.

2. При а $\rightarrow 0$ для решений, удовлетворяющих свойствам (4), (6), скалярное произведение имеет предел.

ЗАмечание 4. Для решений (7), не удовлетворяющих свойству (6), скалярное произведение при $a \rightarrow 0$ расходится. Данный факт аналогичен расходимостям Штюкельберга в квантовой теории поля [10].

Таким образом, применение метода включения и выключения взаимодействия позволяет перенормировать модели, которые не могут быть построены обычными методами типа метода самосопряженных расширений. В примерах, перенормировка которых строится традиционными методами (потенциал типа $\delta$-функции [2], уравнение Хадсона-Партасарати [11] и т. д.), рассмотренная выше процедура включения взаимодействия дает результат, согласующийся с [2], [11]. При этом можно также рассматривать случай, когда коэффициент при гамильтониане взаимодействия явно зависит от времени.

Рассмотренньй в [3] метод индефинитной метрики также укладывается в данную схему. Отметим, что в рассмотренном здесь случае необходимо не только вводить индефинитную метрику, но и расширить пространство состояний, так как контрчлены содержат производные по времени.

Можно ожидать, что аналогичные методы будут полезны и при анализе других моделей, не укладьвающихся в схему [6].

\section{СПИСОК ЦИТИРОВАННОЙ ЛИТЕРАТУРЫ}

1. Боголюбов Н.Н., Ширков Д. В. Введение в теорию квантованных полей. М.: Наука, 1973. 2. Березин Ф. А., Фаддеев Л. Д. // Докл. АН СССР. 1961. Т. 137. № 5. С. 1011. 3. Березин Ф. А. // Матем. сб. 1963. Т. 60. № 4. С. 425. 4. Lee T. // Phys. Rev. 1954. V. 95. № 5. Р. 1329. 5. Челлен Г., Паули В. // УФН. 1956. Т. 60. № 3. С. 425. 6. Кошманенко В. Д. // УМЖ. 1989. Т. 41. №1. С. 3. 7. Хепп К. Теория перенормировок. М.: Наука, 1974. 8. Маслов В. П., Шведов О. Ю. // Матем. заметки. 1998. Т. 63. № 1. С. 147-150. 9. Вольтерра В. Теория функционалов, интегральных и интегро-дифференциальных уравнений. М.: Наука, 1982. 10. Stueckelberg E. C. G. // Phys. Rev. 1951. V. 81. P. 130. 11. Чеботарев А. М. // Матем. заметки. 1996. T. 60. № 5. C. $726-750$. 